\title{
Radiation-Conduction Interaction of Steady Streamwise Surface Temperature Variations on Vertical Free Convection
}

\author{
Md. Kutub Uddin ${ }^{1}$ and Rabindra Nath Mondal ${ }^{2}$ \\ ${ }^{1}$ Department of Mathematics, University of Dhaka, Dhaka 1000, Bangladesh \\ ${ }^{2}$ Mathematics Discipline, Khulna University, Khulna 9208, Bangladesh \\ Correspondence should be addressed to Rabindra Nath Mondal, rnmondal71@yahoo.com
}

Received 11 August 2011; Accepted 21 September 2011

Academic Editors: G. Kyriacou and F. Wang

Copyright (C) 2011 Md. K. Uddin and R. N. Mondal. This is an open access article distributed under the Creative Commons Attribution License, which permits unrestricted use, distribution, and reproduction in any medium, provided the original work is properly cited.

\begin{abstract}
The combined effects of the steady free convective boundary-layer flow induced by a vertical heated surface in the presence of sinusoidal surface temperature variations about a constant mean value with the effect of radiation are examined. The problem is studied using fully numerical techniques. The surface rate of heat transfer eventually alternates in sign with distance from the leading edge, but no separation occurs unless the amplitude of the thermal modulation is sufficiently high. Numerical results are obtained for different values of the physical parameters, the radiation parameter $R_{d}$, the Prandtl number Pr, and the surface temperature wave amplitude $a$. It is found that both the local shear stress and the rate of heat transfer decrease when values of $R_{d}$ increase.
\end{abstract}

\section{Introduction}

It is well known that power-law surface temperature distributions (and also power-law surface heat fluxes) give rise to self-similar boundary layer flows [1,2]. Various papers have been published which deal with the effects of surface variations; for example, Yao [3] and Moulic and Yao $[4,5]$ have sought to investigate the effects of streamwise surface undulations of free and mixed convection from vertical surfaces held at uniform temperatures. Chiu and Chou [6], Hossain et al. [7], and Kim [8] extended these analyses to micropolar fluids, magnetohydrodynamic convection, and non-Newtonian convection, respectively. In a series of papers, Rees and Pop [9-12] and Rees [13] have also considered a large variety of analogous flows in porous media. Of these, only Rees [13] has been concerned with the effect of sinusoidal surface temperature variations, although in that case the surface variations were spanwise, thereby giving rise to a three-dimensional flow field. In the present paper, we are interested in another form of surface variation, namely, sinusoidal variations about 
a mean temperature which is held above the ambient temperature of the fluid. As by Rees [14], this type of surface distribution may be taken as a simplified model of the effects of a periodical array of heaters behind or within the heated surface, an accurate analysis of such a configuration requires a detailed investigation of the effects of solid conduction within the heated surface, but the aim of the present work is to simplify the problem by imposing a surface temperature distribution. In this way, we can determine a large amount of information about the resulting flow using the numerical techniques.

Radiative convective flows are encountered in many industrial and environmental processes, for example, heating and cooling chambers, fossil fuel combustion energy processes, evaporation from large open water reservoirs, astrophysical flows, solar power technology, and space vehicle re-entry. Mathematically, the equations for radiative heat transfer with absorption, scattering, and emission can be generated by one of two approaches, namely, the continuum model or the spectral radiative treatment of a single particle. Details of the derivation of the general equation of radiative heat transfer are provided in the classic monograph by Chandrasekhar [15].

Little is currently known about the boundary layer flows of radiating fluids. The inclusion of conduction-radiation effects in the energy equation, however, leads to a more highly nonlinear partial differential equation. The majority of studies concerned with the interaction of thermal radiation and natural convection were made by Sparrow and Cess [2], Cess [16], Arpaci [17], Cheng and Ozisik [18], Hasegawa et al. [19, 20], and Bankston et al. [21] for the case of a vertical semi-infinite plate. Recently, the problem of natural convectionradiation interaction on boundary layer flows with the Rosseland diffusion approximation has been studied by Hossain and Alim [22] and Hossain et al. [7]. Hossain and Rees [23] investigated the effect of radiation-conduction interaction in the mixed convective flow along a slender impermeable vertical cylinder. The inclusion of radiation terms is complicated and the resulting equations are very difficult to solve. Grief et al. [24] showed that in the optically thin limit, the physical situation can be simplified and they derived an exact solution of the problem of fully developed radiating laminar convection flow in an infinite vertical heated channel. Their analysis was based on the work by Cogley et al. [25]. In the optically thin limit, the fluid does not absorb its own emitted radiation but the fluid absorbs the radiation emitted by the boundaries. It was shown by Cogley et al. [25] that in optically thin limit for a gray-gas near equilibrium, the following relation holds:

$$
\frac{\partial q_{r}}{\partial y}=4\left(T-T_{w}\right) \int_{0}^{\infty} \kappa_{\lambda w}\left(\frac{\partial e_{b \lambda}}{\partial T}\right)_{w} d \lambda=4\left(T-T_{w}\right) I, \quad \text { where } I=\int_{0}^{\infty} \mathcal{\kappa}_{\curlywedge \downarrow}\left(\frac{\partial e_{b \lambda}}{\partial T}\right)_{w} d \lambda
$$

Here $q_{r}$, is the radiative flux, $\kappa_{\curlywedge}$ is the absorption coefficient, $e_{b \lambda}$ is the Planck function, and the subscript $w$ represents the value of a quantity at the wall. Further simplification may be made concerning the spectral properties of radiating gases, but this is not essential for the present analysis. It should be mentioned that Soundalgekar [26] considered the radiative-free convective flow of an optically thin grey-gas past a semi-infinite vertical plate. However, the Rosseland model is valid for isotropic local intensity, and high optical density of the medium and the radiative heat flux is given by [27]

$$
q_{r}=-\frac{16 \sigma \nabla T^{3}}{3\left(a_{R}+\sigma_{\mathrm{s}}\right)} \nabla T,
$$




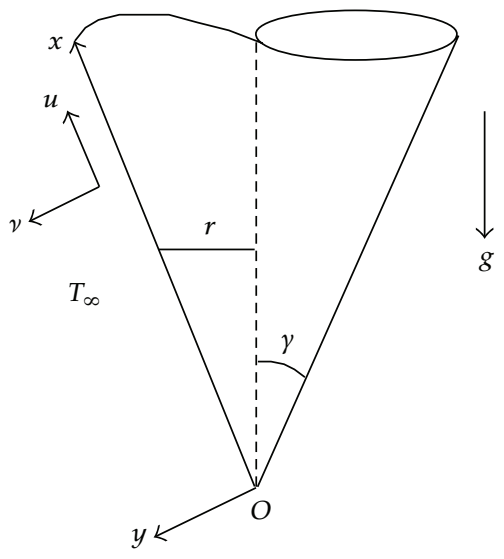

Figure 1: Physical model and the coordinate system.

where $T$ denotes the temperature, $a_{R}$ is the Rosseland absorption coefficient, $\sigma_{s}$ is the scattering coefficient, and $\sigma$ is the Stefan-Boltzmann constant. The thermal boundary-layer equation can be written as

$$
\rho C_{p}\left(u \frac{\partial T}{\partial x}+v \frac{\partial T}{\partial y}\right)=\frac{\partial}{\partial y}\left[\left(\frac{16 \sigma T^{3}}{3 a_{R}}+\kappa\right) \frac{\partial T}{\partial y}\right]
$$

The present paper considers, in detail, how the combined effects of surface radiation and sinusoidal surface temperature profiles in the streamwise direction modify the otherwise self-similar boundary-layer flow. Solutions are presented in terms of the surface rate of heat transfer and shear stress and detailed isotherms are also given. An important feature of the flow is that a near-wall layer develops at large distances downstream of the leading edge. The numerical evidence suggests that this inner layer decreases in thickness with distance downstream. A finite difference method is employed in obtaining the numerical solutions. In this paper, we investigate the combined effects of surface temperature variations and radiation on the steady boundary-layer flow of a Newtonian fluid from a heated vertical surface. The effects of varying different physical parameters on the local skin-friction and local rate of heat transfer are also presented.

\section{Governing Equations and Boundary-Layer Analysis}

A steady two-dimensional laminar-free convection flow of the boundary layer induced by a heated semi-infinite surface immersed in an incompressible Newtonian fluid is considered. In particular, the heated surface is maintained at the steady temperature, fluid having temperature, $T$, from a vertical cone. The physical coordinates $(x, y)$ are chosen such that $x$ is measured from the leading edge in the stream-wise direction and $y$ is measured normal to the surface of the cone. The coordinate system, velocity direction, and the gravity orientation are shown in Figure 1.

The boundary layer form of the equations for flow is

$$
T=T_{\infty}+\left(T_{w}-T_{\infty}\right)(1-a \sin (\pi \hat{x} d)),
$$


where $T_{\infty}$ is the ambient fluid temperature, $T_{w}$ is the mean-surface temperature which is such that $T_{w}>T_{\infty}, a$ is the relative amplitude of the surface temperature variations, and $2 d$ is the wavelength of the variations. After a suitable nondimensionalization, the steady twodimensional equations of motion are given by

$$
\begin{gathered}
u_{x}+v_{y}=0 \\
u u_{x}+v u_{y}=-p_{x}+\mathrm{Gr}^{-1 / 2}\left(u_{x x}+u_{y y}\right)+\theta \\
u v_{x}+v v_{y}=-p_{y}+\mathrm{Gr}^{-1 / 2}\left(v_{x x}+v_{y y}\right)+\theta \\
u \theta_{x}+v \theta_{y}=\alpha \mathrm{Gr}^{-1 / 2}\left[\theta_{y y}+\frac{16 \sigma}{3 \kappa\left(a+\sigma_{s}\right)}\left\{\theta^{3} \theta_{y}\right\}_{y}\right] \\
\theta=\frac{T-T_{\infty}}{T_{w}-T_{\infty}}
\end{gathered}
$$

where Gr is the Grashof number and $\sigma$ is the Prandtl number. In the derivation of (2.2), the Boussinesq approximation has been assumed. We note that the Grashof number has been based on $d$, half the dimensional wavelength of the thermal waves. In the equations, $u$ and $v$ are, respectively, the velocity components in the $x$ and $y$ directions, $T$ is the fluid temperature, $v$ is the kinematic viscosity, $\beta$ is the thermal expansion coefficient, $\alpha$ is the thermal diffusivity, $\kappa$ is the thermal conductivity, $a$ is the Rosseland mean absorption coefficient, $\sigma$ is the StephanBoltzman constant, and $\sigma_{s}$ is the scattering coefficient.

When the surface temperature is uniform and the Grashof number is very large, the resulting boundary-layer flow is self-similar. But the presence of sinusoidal surface temperature distributions, such as that given by (2.1), renders the boundary-layer flow nonsimilar. The boundary-layer equations are obtained by introducing the scaling:

$$
\begin{gathered}
u=u^{*}, \quad v=\mathrm{Gr}^{-1 / 4} v^{*}, \quad x=x^{*}, \\
y=\mathrm{Gr}^{-1 / 4} y^{*}, \quad p=\mathrm{Gr}^{-1 / 2} p^{*}, \quad \theta=\theta^{*},
\end{gathered}
$$

into (2.2), formally letting Gr become asymptotically large and retaining only the leading order terms. Thus, we obtain

$$
\begin{gathered}
u_{x}+v_{y}=0 \\
u u_{x}+v u_{y}=u_{y y}+\theta \\
u v_{x}+v v_{y}=-p_{y}+v_{y y} \\
u \theta_{x}+v \theta_{y}=\alpha \mathrm{Gr}^{-1 / 2}\left[\theta_{y y}+\frac{16 \sigma}{3 \kappa\left(a+\sigma_{s}\right)}\left\{\theta^{3} \theta_{y}\right\}_{y}\right]
\end{gathered}
$$

where the asterisk superscripts have been omitted for clarity of presentation. Equation (2.9) serves to define the pressure field in terms of the two velocity components and is decoupled 
from the other three equations. Therefore, we should not consider it further. As the equations are two-dimensional, we define a stream function $\psi$ in the usual way:

$$
u=\psi_{y}, \quad v=-\psi_{x}
$$

and, therefore, (2.7) is satisfied automatically. Guided by the familiar self-similar form corresponding to a uniform surface temperature, we use the substitution

$$
\psi=x^{3 / 4} f(\eta, x), \quad \theta=g(\eta, x)
$$

where

$$
\eta=\frac{y}{x^{1 / 4}}
$$

is the pseudosimilarity variable. Equations (2.8) and (2.9) reduce to

$$
\begin{gathered}
f^{\prime \prime \prime}+g+\frac{3}{4} f f^{\prime \prime}-\frac{1}{2} f^{\prime} f^{\prime}+x\left(f_{x} f^{\prime \prime}-f_{x} f^{\prime}\right)=0, \\
\frac{1}{\operatorname{Pr}}\left[\left\{1+\frac{4}{3} R_{d}\left(1+\left(\theta_{w}-1\right) g\right)^{3}\right\} g^{\prime}\right]^{\prime}+\frac{3}{4} f g^{\prime}+x\left(f_{x} g^{\prime}-f^{\prime} g_{x}\right)=0,
\end{gathered}
$$

and the boundary conditions are

$$
\begin{aligned}
& f=0, \quad f^{\prime}=0, \quad g=1+a \sin \pi x, \\
& \text { at } \eta=0, \quad f^{\prime} g \longrightarrow 0 \quad \text { as } \eta \longrightarrow \infty .
\end{aligned}
$$

In (2.14)-(2.16), primes denote derivatives with respect to $\eta$.

\section{Numerical Solutions}

The parabolic system of $(2.14)-(2.16)$ is nonsimilar and its numerical solution must be obtained using a marching method. The results presented here were obtained using the Keller-box method. After reducing (2.14)-(2.15) to first-order form in $\eta$, the subsequent second-order accurate discretisation based halfway between the grid points in both the $\eta$ and $x$-directions yields a set of nonlinear difference equations which are solved using a multidimensional Newton-Raphson iteration scheme. The results presented in Figures 2-5 are based on uniform grids in both coordinate directions. There were 201 grid points lying between $\eta=0$ and $\eta=20$ and 401 between $x=0$ and $x=20$. We restrict the presentation of our results to the two values of the Prandtl number, $\operatorname{Pr}=0.7$ (air) and $\operatorname{Pr}=7.0$ (water).

Figure 2(a) shows the evolution of $f^{\prime \prime}(\eta=0)$ with $x$, a scaled surface shear stress, for various values of the temperature wave amplitude, $a$, and the constant radiation parameter $R_{d}$ for $\operatorname{Pr}=0.7$. The corresponding rates of surface heat transfer are shown in Figure 2(b). Some aspects of the overall behavior of these curves may be explained by observing that 


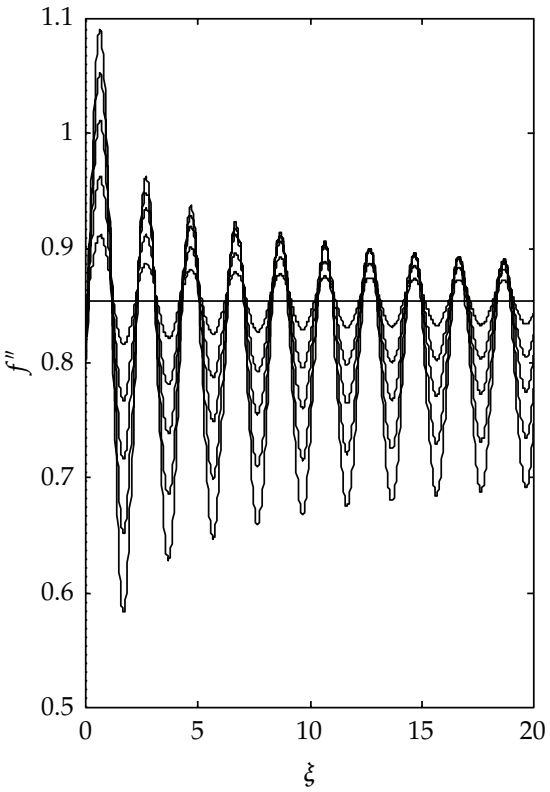

(a)

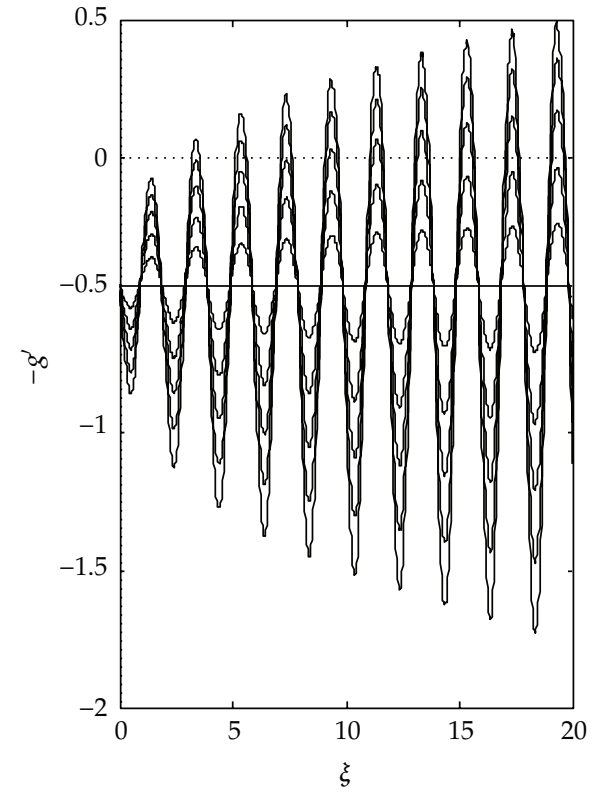

(b)

Figure 2: (a) Skin friction and (b) rate of heat transfer against $\xi$ for $a=0.0,0.2,0.4,0.6,0.8,1.0$ at $\operatorname{Pr}=0.7$.

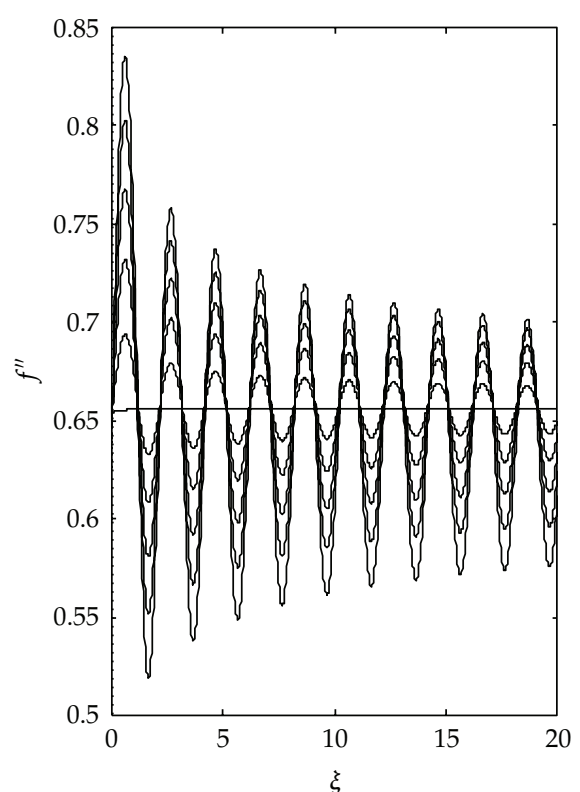

(a)

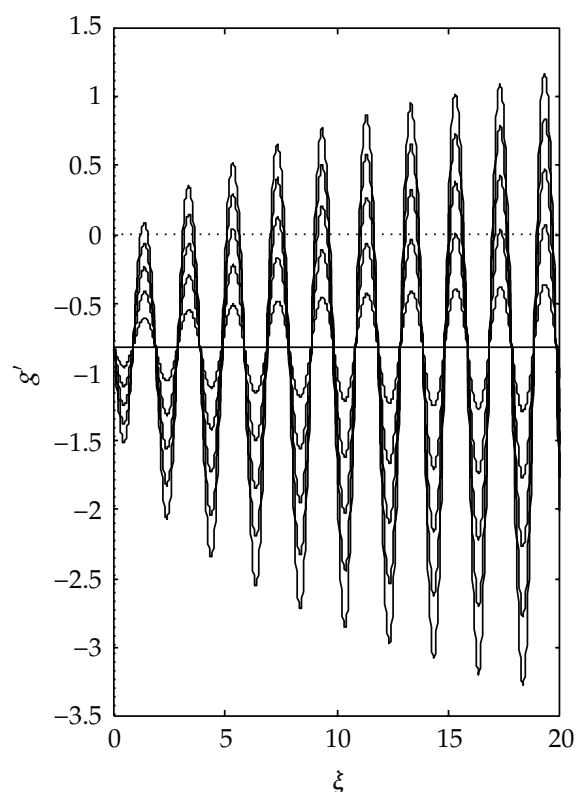

(b)

Figure 3: (a) Skin friction and (b) rate of heat transfer against $\xi$ for $a=0.0,0.2,0.4,0.6,0.8,1.0$ for $\operatorname{Pr}=7.0$. 


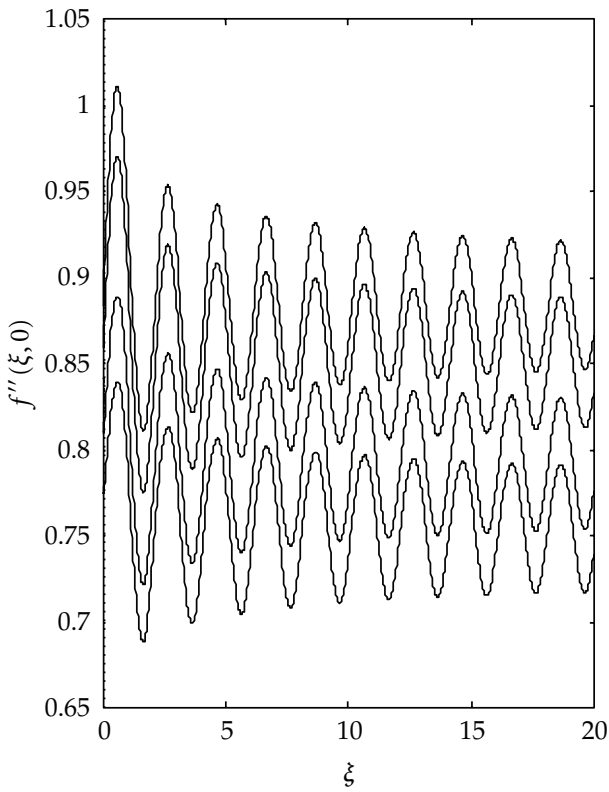

(a)

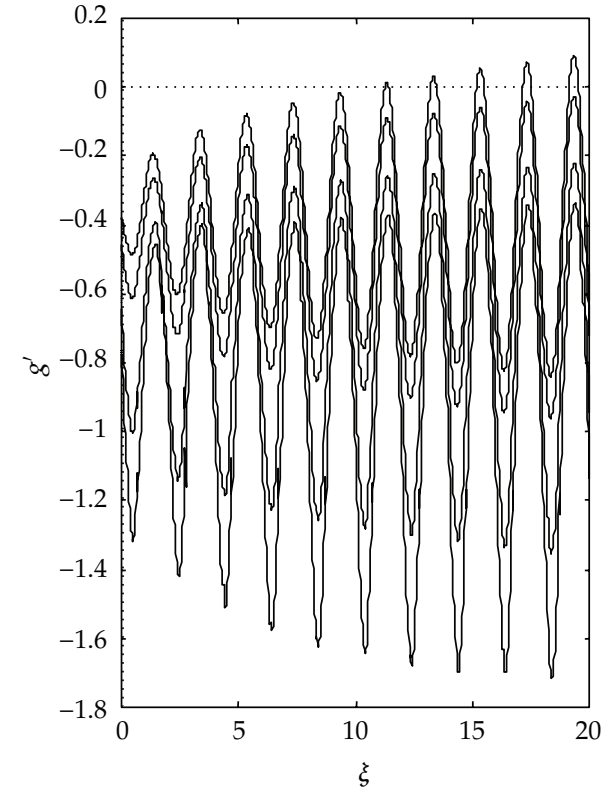

(b)

Figure 4: (a) Skin friction and (b) rate of heat transfer against $\xi$ for $R_{d}=0.0,1.0,5.0,10$ for $\operatorname{Pr}=0.7$.

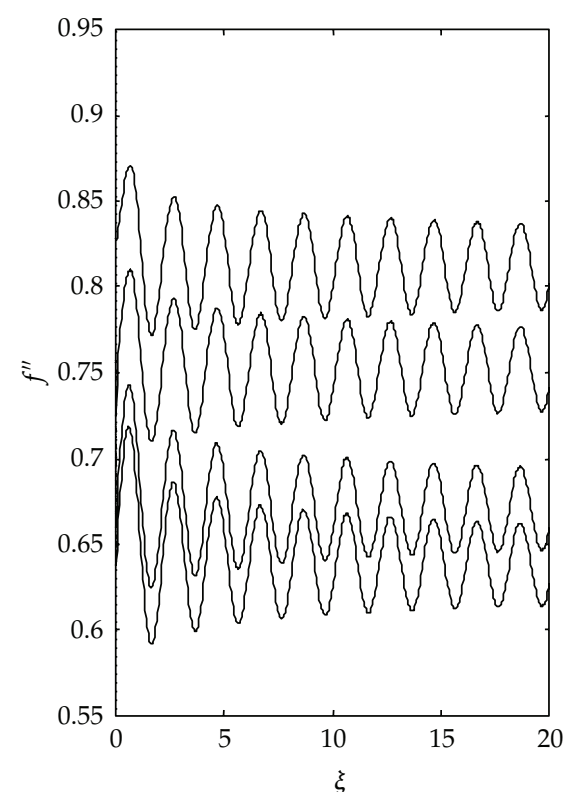

(a)

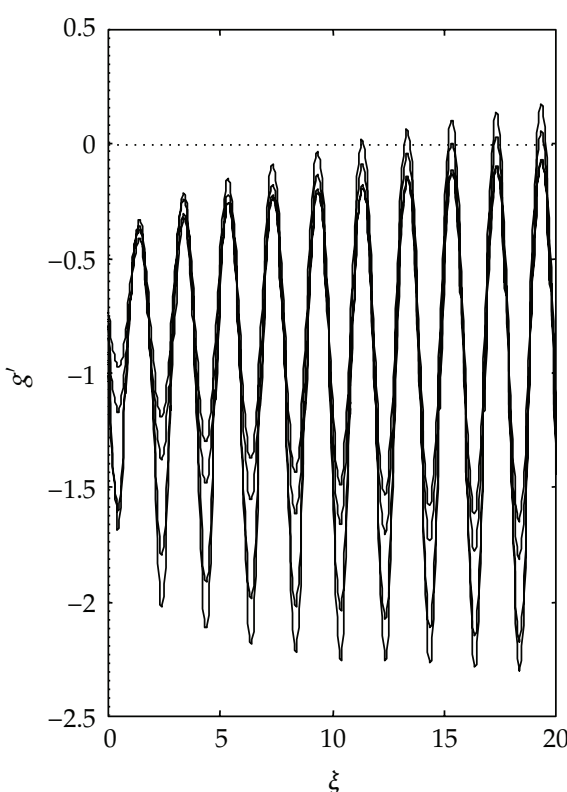

(b)

Figure 5: (a) Skin friction and (b) rate of heat transfer against $\xi$ for $R_{d}=0.0,1.0,5.0,10$ for $\operatorname{Pr}=7.0$. 
the boundary layer is thinner when the surface temperature is relatively high and thicker when it is low. This arises because relatively high surface temperatures induce relatively large upward fluid velocities with the consequent increase in the rate of entertainment into the boundary layer. This causes, in turn, a thinning of the boundary layer. Thus, we should expect high shear stresses and rates of heat transfer at, or perhaps just beyond, where the surface temperature attains its maximum values. There is an obvious qualitative difference between the curves shown in Figure 2(a) and those in Figure 2(b). It is seen that as $x$ increases, the amplitude of oscillation of the shear stress curves decays slowly, whereas the amplitude of heat transfer curves increases with $x$. Indeed, the curves in Figure 2(b) suggest that, whatever the value of $a$ is, there will always be a value of $x$ beyond which some part of the rate of the heat transfer curve between successive surface temperature maxima will be positive. This somewhat unusual phenomenon for boundary layer flows may be explained by noting that when relatively hot fluid encounters a relatively cold part of the heated surface, the overall heat transfer will occur from the fluid into the surface rather than the other way around.

Figure 3(a) shows the surface shear stress for various values of the temperature wave amplitude $a$ and constant radiation parameter $R_{d}$ for $\operatorname{Pr}=7.0$. The corresponding rates of surface heat transfer are shown in Figure 3(b). The same result, as shown above, is also happening in this case. Besides this, shear stress and rates of surface heat transfer are decreasing at $\operatorname{Pr}=7.0$ than that at $\operatorname{Pr}=0.7$. Figure 4(a) presents surface shear stress for various values of radiation parameter $R_{d}$ and constant temperature wave amplitude $a$ for $\operatorname{Pr}$ $=0.7$. The corresponding rates of surface heat transfer are shown in Figure $4(\mathrm{~b})$. This figure describes that when the radiation parameter is increasing both the shear stress and rates of surface heat transfer are decreasing. It might be mentioned here that whenever the value of radiation parameter $R_{d}=0.0$, that is, when there is no effect of radiation, then these results have an excellent agreement with the results of Rees [14]. Figure 5(a) shows surface shear stress for various values of radiation parameter $R_{d}$ and constant temperature wave amplitude $a$ for $\operatorname{Pr}=7.0$. The corresponding rates of surface heat transfer are shown in Figure 5(b). Here, we observe that both the shear stress and rates of surface heat transfer are decreasing significantly for $\operatorname{Pr}=7.0$ than that for $\operatorname{Pr}=0.7$.

In Figure 6, we show the isotherms for $\operatorname{Pr}=0.7$ and $a=0.2$ for $R_{d}=0.0,5.0$, and 10.0. Here, we see that the boundary layer maintains its overall thickness in terms of $\eta$ when $x$ is large, although variations in thickness are clearly presented when $x$ is small. The thickness of the region, in which strong surface, induced temperature variations are present, reduces slowly in size as $x$ increases. It is also clear that isolines are dense in the areas of high values (e.g., high temperature) and sparse in the areas with low intensity.

In Table 1, we show shear stress and surface rate of heat transfer for different values of Pr when $R_{d}=0.0$ and 5.0. As seen in Table 1, both the shear stress and the rate of surface heat transfer are decreasing due to increasing the value of $\operatorname{Pr}$ as well as radiation parameter $R_{d}$. Moreover, when $R_{d}=0.0$, that is, when there is no radiation, these results have an excellent agreement with the result found by Rees [14].

\section{Conclusions}

In this paper, the combined effects of radiaton-conduction interaction with steady streamwise surface temperature variations on vertical free convection have been investigated numerically by using a finite difference method. The effects of variations in the surface temperature wave amplitude and the Prandtl number on the shear stress and rate of surface heat transfer have been given. 


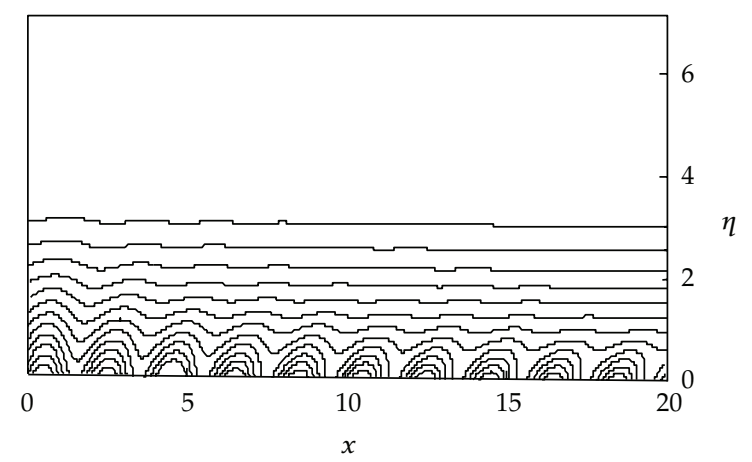

(a)

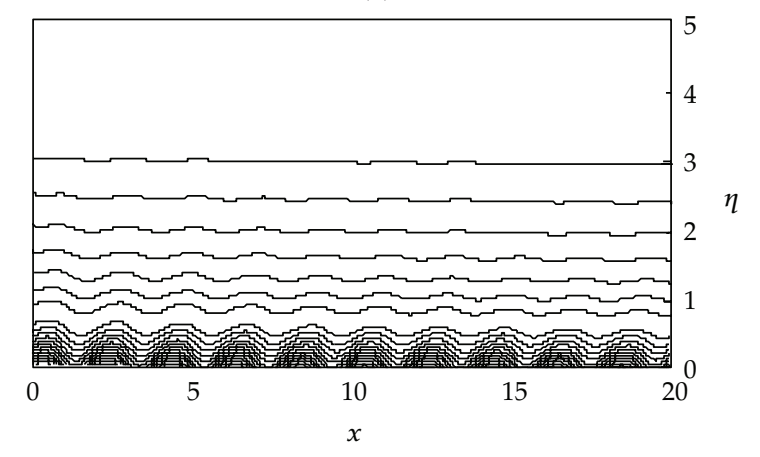

(b)

Figure 6: Isotherms for $\operatorname{Pr}=0.7$ and $a=0.2$. (a) $R_{d}=0.0$, (b) $R_{d}=10.0$.

Table 1: Shear stress and surface rate of heat transfer for different values of $\operatorname{Pr}$ when $R_{d}=0.0$ and 5.0.

\begin{tabular}{lcccc}
\hline $\operatorname{Pr}$ & $f^{\prime \prime}$ & & & $g^{\prime}$ \\
\hline 0.01 & $R_{d}=0.0$ & $R_{d}=5.0$ & $R_{d}=0.0$ & $R_{d}=5.0$ \\
0.02 & 1.39476 & 0.12113 & -0.05752 & -4.88760 \\
0.05 & 1.35574 & 0.11860 & -0.07897 & -5.13263 \\
0.10 & 1.28438 & 0.11860 & -0.12000 & -5.13533 \\
0.20 & 1.21504 & 0.11861 & -0.16274 & -5.13626 \\
0.50 & 1.13287 & 0.11862 & -0.21772 & -5.13635 \\
0.70 & 1.00855 & 0.11863 & -0.31195 & -5.13604 \\
1.00 & 0.96012 & 0.11863 & -0.35320 & -5.13553 \\
2.00 & 0.11864 & -0.40103 & -5.13496 \\
3.00 & 0.90819 & 0.11865 & -0.50661 & -5.13438 \\
4.00 & 0.80789 & 0.11865 & -0.57667 & -5.13386 \\
5.00 & 0.75077 & 0.11866 & -5.13340 \\
6.00 & 0.71129 & 0.11866 & -0.63042 & -5.13301 \\
7.00 & 0.68136 & 0.11866 & -0.71234 & -5.13269 \\
8.00 & 0.65740 & 0.11867 & -0.74551 & -5.13245 \\
10.0 & 0.63751 & 0.11867 & -0.77519 & -5.13226 \\
20.0 & 0.62056 & -0.82684 & -5.13214 \\
40.0 & 0.59284 & 0.11867 & -1.00519 & -5.13215 \\
70.0 & 0.51190 & 0.11866 & -1.21461 & -5.13247 \\
100.0 & 0.43921 & 0.11865 & -1.41044 & -5.13332 \\
\hline & 0.38665 & 0.11862 & -1.54955 & -5.13484 \\
\hline
\end{tabular}


We calculated the surface shear stress (skin friction) and rate of heat transfer for various values of the temperature wave amplitude at a constant radiation parameter for the Prandtl numbers $\operatorname{Pr}=0.7$ and $\operatorname{Pr}=7.0$. It is seen that as $x$ increases, the amplitude of oscillation of the shear stress curves decays slowly, whereas the amplitude of heat transfer curves increases with $x$. We then calculated the surface shear stress (skin friction) and the rate of heat transfer for various values of radiation parameter at a constant temperature wave amplitude for $\operatorname{Pr}=0.7$ and $\operatorname{Pr}=0.7$ and it is found that when the radiation parameter is increasing both the shear stress and rates of surface heat transfer are decreasing, and at $R_{d}=0.0$, that is, when there is no effect of radiation, the results have an excellent agreement with the results of Rees [14]. It is also found that both the shear stress and rates of surface heat transfer are decreasing significantly for $\operatorname{Pr}=0.7$ than that at $\operatorname{Pr}=0.7$.

\section{Nomenclature}

a: Surface temperature wave amplitude

$a_{R}:$ Rosseland mean absorption coefficient

$f$ : Dimensionless velocity function

g: Dimensionless temperature acceleration due to gravity

Gr: Grashof number

Pr: Prandtl number

$d: \quad$ Half the dimensional thermal wave length

$R_{d}$ : Radiation parameter

$p$ : Pressure

$u, v$ : Velocity components

$T$ : $\quad$ Temperature of the fluid

$T_{\infty}$ : Temperature of the ambient fluid

$x$ : Streamwise and cross stream

$y$ : Cartesian coordinate

$\psi:$ Stream function

$\sigma: \quad$ Stefan-Boltzman constant

$\sigma_{s}:$ Scattering coefficient

$\beta$ : Coefficient expansion

$v$ : Kinematic coefficient of viscosity

$\eta$ : Psuedo-similarity variable

$\theta: \quad$ Temperature

$\theta_{w}:$ Surface Temperature parameter

$\xi: \quad$ Dimensionless $x$ coordinates

$\rho$ : Density of the fluid

$T_{w}$ : Mean-surface temperature to the wall.

\section{References}

[1] S. Ostrach, "An analysis of laminar free convection flow and heat transfer about a flat plate parallel to the direction of the generating body force," Technical note number A084083, National Association for Campus Activities (NACA IV), 1952.

[2] E. M. Sparrow and R. D. Cess, "Radiation Heat Transfer," International Journal of Heat and Mass Transfer, vol. 5, pp. 179-806, 1962.

[3] L. S. Yao, "Natural convection along a vertical wavy surface," Journal of Heat Transfer, vol. 105, no. 3, pp. 465-468, 1983. 
[4] S. G. Moulic and L. S. Yao, "Mixed convection along a wavy surface," Journal of Heat Transfer, vol. 111, pp. 974-979, 1989.

[5] S. G. Moulic and L. S. Yao, "Natural convection along a vertical wavy surface with uniform heat flux," Journal of Heat Transfer, vol. 111, pp. 1106-1108, 1989.

[6] C. P. Chiu and H. M. Chou, "Free convection in the boundary layer flow of a micropolar fluid along a vertical wavy surface," Acta Mechanica, vol. 101, no. 1-4, pp. 161-174, 1993.

[7] M. A. Hossain, M. A. Alim, and H. S. Takhar, "Mixed convection boundary layer flow along a vertical cylinder," Journal of Applied Mechanics and Engineering, vol. 20, pp. 117-122, 1998.

[8] E. Kim, "Natural convection along a wavy vertical plate to non-Newtonian fluids," International Journal of Heat and Mass Transfer, vol. 40, no. 13, pp. 3069-3078, 1997.

[9] D. A. S. Rees and I. Pop, “A Note on free convection along a vertical wavy surface in a porous medium," Journal of Heat Transfer, vol. 116, no. 2, pp. 505-508, 1994.

[10] D. A. S. Rees and I. Pop, "Free convection induced by a horizontal wavy surface in a porous medium," Fluid Dynamics Research, vol. 14, no. 4, pp. 151-166, 1994.

[11] D. A. S. Rees and I. Pop, "Non-Darcy natural convection from a vertical wavy surface in a porous medium," Transport in Porous Media, vol. 20, no. 3, pp. 223-234, 1995.

[12] D. A. S. Rees and I. Pop, "The effect of longitudinal surface waves on free convection from vertical surfaces in porous media," International Communications in Heat and Mass Transfer, vol. 24, no. 3, pp. 419-425, 1997.

[13] D. A. S. Rees, "Three-dimensional free convection boundary layers in porous media induced by a heated surface with spanwise temperature variations," Journal of Heat Transfer, vol. 119, no. 4, pp. 792-798, 1997.

[14] D. A. S. Rees, "The effect of steady streamwise surface temperature variations on vertical free convection," International Journal of Heat and Mass Transfer, vol. 42, no. 13, pp. 2455-2464, 1999.

[15] S. Chandrasekhar, Radiative Heat Transfer, Dover, New York, NY, USA, 1960.

[16] R. D. Cess, "The interaction of thermal radiation with free convection heat transfer," International Journal of Heat and Mass Transfer, vol. 9, no. 11, pp. 1269-1277, 1966.

[17] V. S. Arpaci, "Effect of thermal radiation with free convection from a heated vertical plate," International Journal of Heat and Mass Transfer, vol. 15, pp. 1243-1252, 1968.

[18] E. H. Cheng and M. N. Ozisik, "Radiation with free convection in an absorbing, emitting and scattering medium," International Journal of Heat and Mass Transfer, vol. 15, no. 6, pp. 1243-1252, 1972.

[19] S. Hasegawa, R. Echigo, and K. Fakuda, "Analytic and experimental studies on simultaneous radiative and free convective heat transfer along a vertical plate," Journal of the Japan Society of Mechanical Engineers, vol. 38, no. 315, pp. 2873-2883, 1972.

[20] S. Hasegawa, R. Echigo, and K. Fakuda, "Analytic and experimental studies on simultaneous radiative and free convective heat transfer along a verticalplate," Journal of the Japan Society of Mechanical Engineers, vol. 39, no. 317, pp. 250-257, 1973.

[21] J. D. Bankston, J. R. Lloyd, and J. L. Novotny, "Radiation convection interaction in an absorbingemitting liquid in natural convection boundary layer flow," Journal of Heat Transfer, vol. 99, no. 1, pp. 125-127, 1977.

[22] M. A. Hossain and M. A. Alim, "Natural convection-radiation interaction boundary layer flow along a thin vertical cylinder," Heat and Mass Transfer/Waerme- und Stoffuebertragung, vol. 32, no. 6, pp. 515520, 1997.

[23] M. A. Hossain and D. A. S. Rees, "Radiation-conduction interaction on mixed convection flow along a slender vertical cylinder," Journal of Thermophysics and Heat Transfer, vol. 12, no. 4, pp. 611-614, 1998.

[24] R Greif, I. S. Habib, and J. C. Lin, "Laminar free convection of a radiating gas in vertical channel," The Journal of Fluid Mechanics, vol. 46, pp. 513-520, 1971.

[25] A. C. Cogley, W. G. Vincenti, and S. E. Giles, "Differential approximation for radiative in a non-gray gas near equilibrium," The American Institute of Aeronautics and Astronautics Journal, vol. 6, pp. 551-553, 1968.

[26] V. M. Soundalgekar, "Radiative convective flow past a semi-infinite vertical plate," Modeling, Measurement and Control: B, vol. 51, no. 2, pp. 31-40, 1993.

[27] R. Siegel and J. R. Howell, Thermal Radiation Heat Transfer, McGraw-Hill, New York, NY, USA, 1987. 


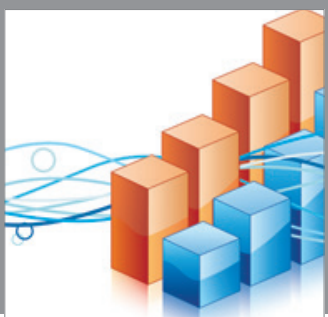

Advances in

Operations Research

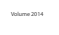

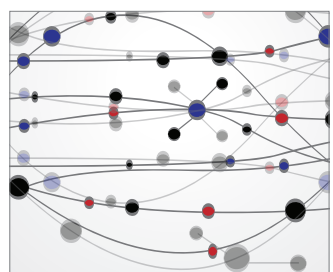

\section{The Scientific} World Journal
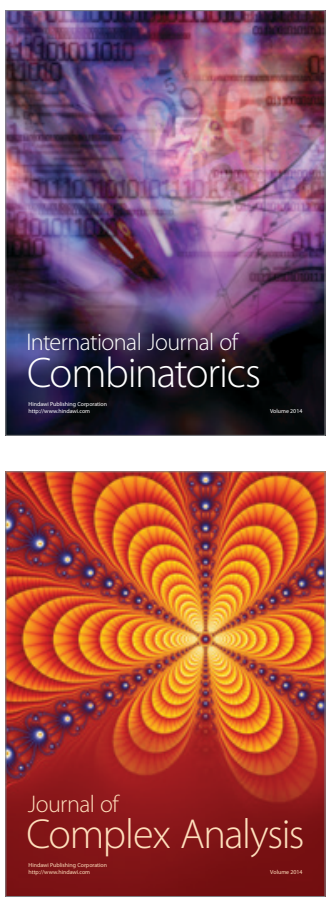

International Journal of

Mathematics and

Mathematical

Sciences
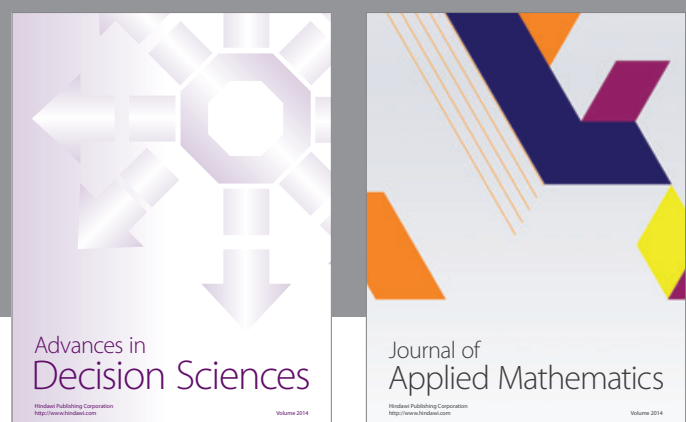

Journal of

Applied Mathematics
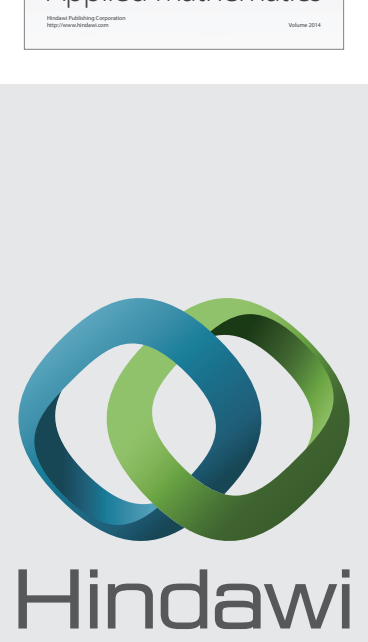

Submit your manuscripts at http://www.hindawi.com
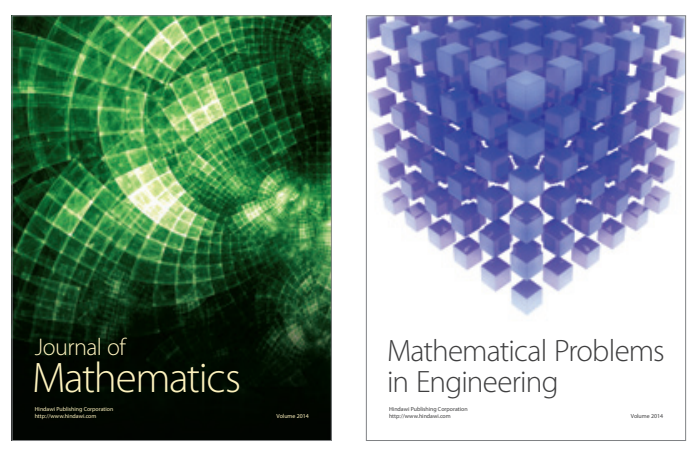

Mathematical Problems in Engineering
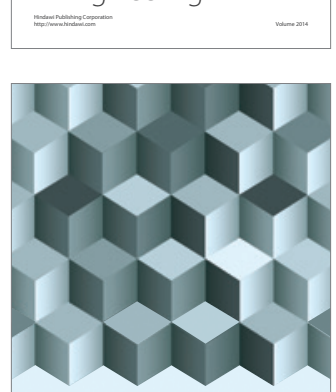

Journal of

Function Spaces
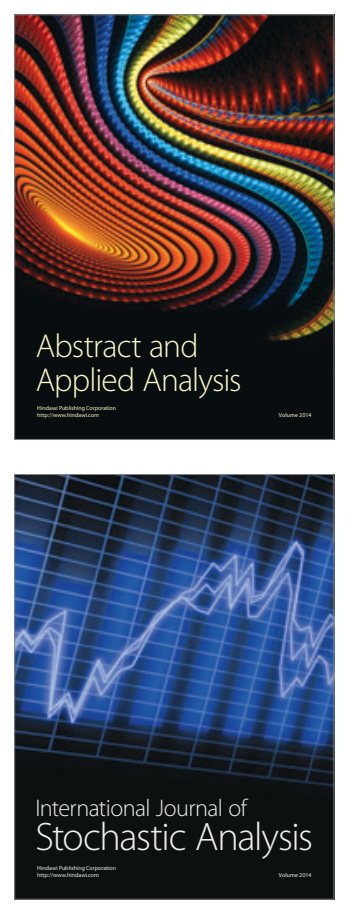

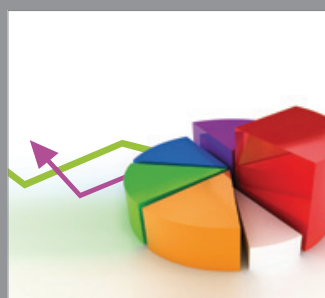

ournal of

Probability and Statistics

Promensencen
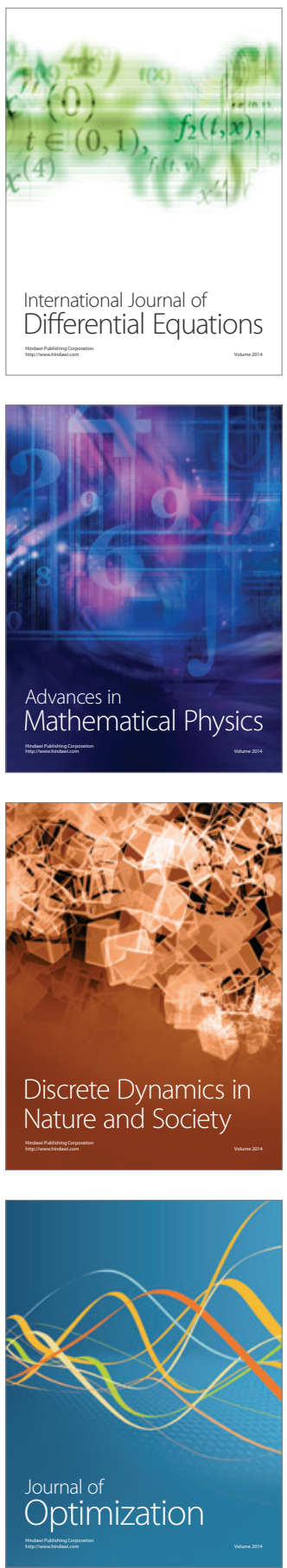